\title{
Numerical modelling of the surface cracking tendency in solidifying ceramic materials
}

\author{
J. Dobrovská ${ }^{1}$, F. Kavička ${ }^{2}$, K. Stránský ${ }^{2} \&$ B. Sekanina ${ }^{2}$ \\ ${ }^{1}$ Faculty of Metallurgy and Materials Engineering, \\ VSB-Technical University of Ostrava, Czech Republic \\ ${ }^{2}$ Faculty of Mechanical Engineering, \\ Brno University of Technology, Czech Republic
}

\begin{abstract}
EUCOR is a ceramic material resistant to extreme heat and wear. A block made of EUCOR with dimensions of $350 \times 200 \times 400 \mathrm{~mm}$ was used for the numerical calculation and the experiment. The numerical model of the casting block temperature field determines the temperature gradients, the solidification rate and the local solidification time (i.e., the time between the liquidus and solidus temperatures). The local solidification time significantly affects the forming of the pouring structure of the given material and it makes one of the input parameters for the second cooperating chemical heterogeneity model. This model and its application on EUCOR samples prove that the applied measuring method of the chemical heterogeneity provides detailed quantitative information on the material structure and makes possible the analysis of the solidification process. The analysis of this process entails statistical processing of results of the heterogeneity measurements of the EUCOR components and it performs correlation of individual components during the solidification. The model of the chemical heterogeneity enables estimation of the structure parameters at any critical point of castings. The combining of both models makes it possible to estimate the structure parameter, which expresses the size of the crystallites in the resultant material structure. It is a tool for the estimation of the tendency to surface cracking and fracturing tendency at the critical point of casting made of EUCOR.
\end{abstract}

Keywords: ceramic material, numerical model, solidification, local solidification time, chemical heterogeneity, tendency to cracking. 


\section{Introduction}

The corundo-baddeleyite material EUCOR is heat and wear resistant even at extreme temperatures and it is also corrosion resistant. EUCOR belongs to the not well known system of the $\mathrm{Al}_{2} \mathrm{O}_{3}-\mathrm{SiO}_{2}-\mathrm{ZrO}_{2}$ oxide ceramics. It is produced only in several plants in the world - and in the Czech Republic it is called EUCOR. The production process utilises exclusively the waste material from relined furnaces in glass-manufacturing plants. The melting of this material is done in electric arc furnaces lined with material of the same chemical composition. The basic approximate chemical composition (in wt.\%) is $13-17 \% \mathrm{SiO}_{2}, 49-52 \% \mathrm{Al}_{2} \mathrm{O}_{3}, 30-33 \% \mathrm{ZrO}_{2}, 0.1 \% \mathrm{TiO}_{2}, 0.2 \mathrm{CaO}, 0.2 \% \mathrm{FeO}$, and $(1.0-2.0 \%)$ alkaline oxides. The mineralogical composition, i.e. the phase composition of this ceramic material, is as follows (in wt.\%): $48-50 \%$ corundum, $30-32 \%$ baddeleyite, and $18-20 \%$ glass phase.

From the foundry viewpoint, EUCOR has certain characteristics similar to the behaviour of cast metals, especially steels. For example, the cooling of EUCOR blocks was monitored and measured with the objective to determine the solidification constant $\mathrm{K}\left[\mathrm{cm} \cdot \mathrm{min}^{-1 / 2}\right]$, according to Chvorinov [1]. During the calculation of this constant, according to Chvorinov, the heat transfer was considered only in the direction perpendicular to the wall of the casting, together with the corresponding calculated modulus of the cast blocks $\mathrm{M}=10 \mathrm{~cm}$. This corresponds to the basic relation $\mathrm{M}=\mathrm{K} \sqrt{\mathrm{t}}$, where $t$ is the solidification time in minutes. The solidification constant:

- $\mathrm{K}=0.669\left[\mathrm{~cm} \cdot \mathrm{min}^{-1 / 2}\right]$ for a casting into a mould made of a mixture of sand and water-glass, without metal chills, and

- $\mathrm{K}=0.890\left[\mathrm{~cm} \cdot \mathrm{min}^{-1 / 2}\right.$ ] for a casting into a mould made of a mixture of sand and water-glass with approximately $50 \%$ of metal chills.

The determined solidification constants are close to those of metals.

It is also possible to apply risering to EUCOR castings - to a certain extent in a similar way as casting steels. The surface layer of the oxidic melt solidifies quickly and the crust prevents the melt from the riser to flow to the mould. For this reason, it is necessary to perforate the crust several times during the solidification. Due to the high volume contraction during solidification $(6.5 \%)$, it is necessary to select a riser with the casting-to-riser ratio of 7:3 and count with the $70 \%$ utilization of melt even when the level (of the riser) is insulated with Sibral and its multiple perforations. With risers prone to cracking, it is necessary to ensure the cooling rate of less than $50^{\circ} \mathrm{C} /$ hour [2] within the temperature range from $970^{\circ} \mathrm{C}$ to $560^{\circ} \mathrm{C}$.

The paper introduces a numerical model of the temperature field and a consequent model of the chemical heterogeneity and their application on cast EUCOR.

\section{Numerical model of three-dimensional temperature field}

The solidification, crystallization and cooling are the most important technological processes in the foundry industry. They are cases of three- 
dimensional (3D) heat and mass transfers. In systems comprising the casting, the mould and environs, all three kinds of heat transfer take place. Due to the fact that these problems cannot be solved analytically - even with the second-order partial differential Fourier equation (1) (the assumption: a mass transfer is neglected and the conduction is considered the most important of the three kinds of heat transfer) - it is necessary to apply the numerical methods. Equation (1) describes the transient temperature field in a mould with the assumption that its properties $-k$-heat conductivity, $c$-specific heat capacity and $\rho$-density are constant.

$$
\frac{\partial T}{\partial \tau}=\frac{k}{\rho \cdot c}\left(\frac{\partial^{2} T}{\partial x^{2}}+\frac{\partial^{2} T}{\partial y^{2}}+\frac{\partial^{2} T}{\partial z^{2}}\right)
$$

The Fourier equation for a casting must be adapted in a way that it describes the temperature field of a casting in its all three phases: in the melt, in the mushy zone, and in the solid phase. In this case it is necessary to introduce the specific volume enthalpy $h_{v}=c . \rho . T$, which is dependent on the temperature. The thermodynamic enthalpy function includes also latent heat of phase or structural changes. Under these assumptions equation (1) can be rewritten in the following form:

$$
\frac{\partial h_{v}}{\partial t}=\frac{\partial}{\partial x}\left(k \frac{\partial T}{\partial x}\right)+\frac{\partial}{\partial y}\left(k \frac{\partial T}{\partial y}\right)+\frac{\partial}{\partial z}\left(k \frac{\partial T}{\partial z}\right)
$$

The specific heat capacity $c$, density $\rho$ and heat conductivity $k$ are also the functions of temperature. The enthalpy function must be known for the cast material.

The temperature of the general nodal point of the casting is obtained from the enthalpy-temperature dependence, which must be known for the relevant material (Figure 1).

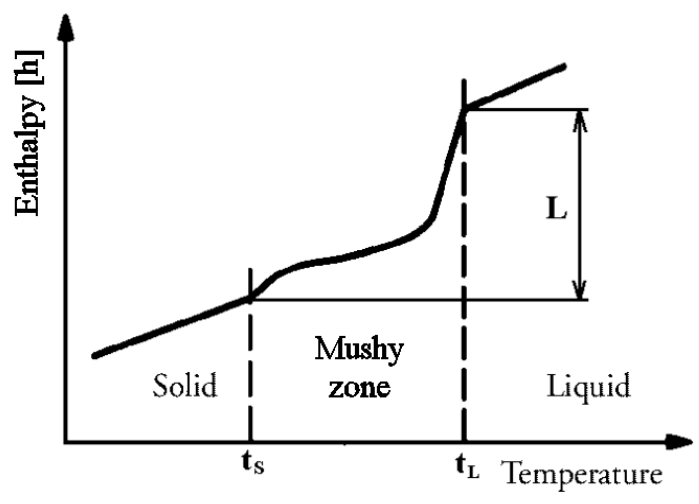

Figure 1: $\quad$ Enthalpy as a function of temperature. 
The explicit differential method was chosen for this examination because it enabled the application of the most convenient method of numerical simulation of the release of latent heat of the phase and the structural changes by using the thermodynamic enthalpy function.

The solution also considers the non-linearity of the task, i.e.:

- The dependence of the thermo-physical properties of all materials entering the system on the temperature, and

- The dependence of the heat-transfer coefficients on the temperature for all boundaries of the system, i.e. for the surfaces of the casting and mould.

Our own proprietary software has integrated an original mesh generator (i.e. pre-processing), as well as the post-processing (i.e. graphical output) of the results. The user can change the pouring parameters, the dimensions of the casting-mould system and the dimensions of the elementary mesh volume before the actual calculation. The number of nodal points can be anywhere within the range from $10^{6}$-to- $10^{7}$ - this density makes it possible to cover castings of more complex shapes, as well as to approximate the linear distribution of temperatures between individual points of the $3 \mathrm{D}$ mesh and even within time intervals.

The exactness of the numerical solution depends not only on the spatial and temporal discretisation, but also on the precision, with which the thermophysical properties of all materials entering the system are determined. In addition, it depends also on precision of derivation of the boundary conditions.

\subsection{Application of the temperature field model}

The application of the 3D numerical model on a transient temperature field requires systematic experimentation, including the relevant measuring of the operational parameters directly in the foundry. The results of the measurements focused on measuring temperatures, serve not only for the evaluation of the exactness of the model, but also for the maintenance of continuity of the procedure: real process (risering, melting, pouring, solidification, etc.) $\rightarrow$ input data $\rightarrow$ numerical analysis $\rightarrow$ optimisation $\rightarrow$ correction of the real process (risering, melting, pouring, solidification, etc.).

Block made of EUCOR with dimensions of $350 \times 200 \times 400 \mathrm{~mm}$ (Figure 2) was used for the numerical calculation and for the experiment. The temperature measurements (using thermocouples) and their consequent confrontation with the calculation proved that it was possible to apply the numerical model on basic calculations of the EUCOR solidification and cooling. It was also possible to determine the temperature gradients, the rate of solidification and the local solidification time (i.e., the time between the liquidus and solidus temperatures at the given point). The local solidification time $\theta$ significantly affected the resulting material structure. 

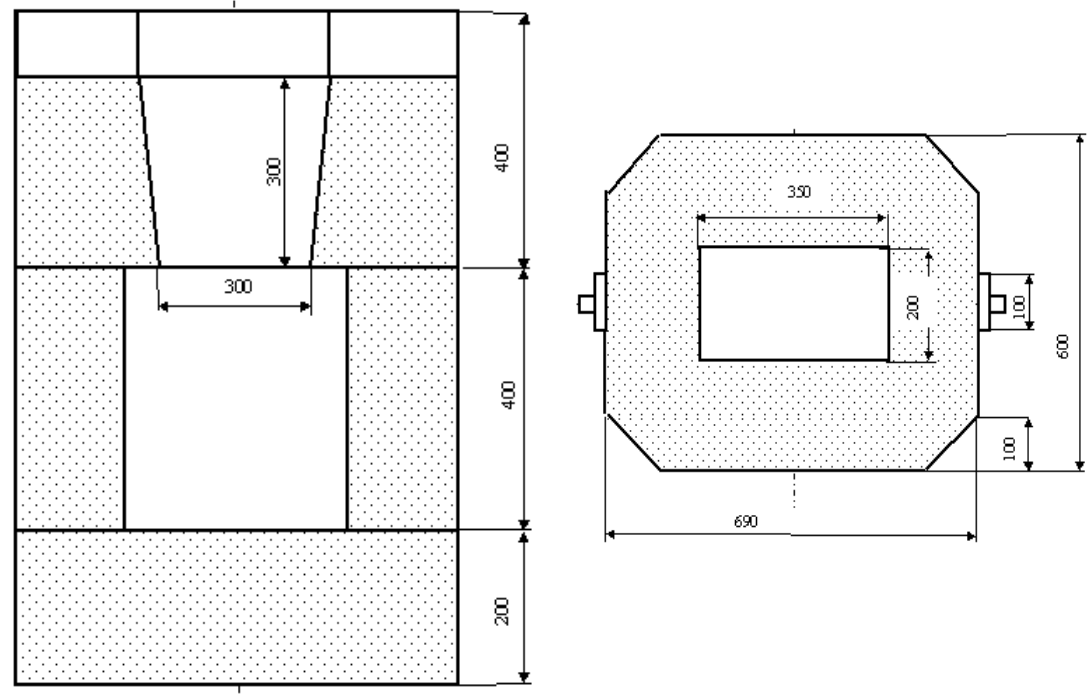

Figure 2: The casting-riser-mould system.

\section{Chemical heterogeneity model}

The principle of the chemical heterogeneity model has been described in detail and explained in the publication concerning the solidification of steels [3]. Its utilisation for the description of the EUCOR chemical heterogeneity is based on the analogy of solidification laws of this material with the solidification of steels. The preconditions for the application of the chemical heterogeneity model for EUCOR are as follows:

The analytical distributions of micro-heterogeneity of the ceramic material oxides are available and also their effective partitioning coefficients are known. It is possible to describe the development of solidification of the ceramic material with the help of analogical models used for the solidification of metal alloys.

If the well-known Brody-Flemings model [4] is applied for the description of the segregation of oxides of the solidifying ceramic material:

$$
C_{s}=k_{e f} C_{0}\left[1-\left(1-2 \alpha k_{e f}\right) g_{s}\right]^{\left(k_{e f}-1\right) /\left(1-2 \alpha k_{e f}\right)},
$$

it is possible to express the relationship between the heterogeneity index $I_{\text {het }}$ ( $I_{\text {het }}$ $=\mathrm{s}_{\mathrm{C}} / \mathrm{C}_{0}$ ) of the relevant oxide, its effective partitioning coefficient $k_{\text {ef }}$ and the dimensionless parameter $\alpha$ by using the following equation

$$
\ln \left(2 \alpha k_{e f}\right) /\left(1-2 \alpha k_{e f}\right)=\left[\ln \left(1+n I_{H}^{(m)}\right) / k_{e f}\right] /\left(k_{e f}-1\right) .
$$

The right side of Eq. (4), based on the measurements of micro-heterogeneity, is already known and it is thus possible to determine the parameter $\alpha$. In 
equations (3) and (4) the individual parameters represent the following: $C_{s}$ [wt. $\%$ ] - the oxide concentration in the solid phase; $C_{0}$ [wt. \%] - arithmetic mean of the oxide concentration of the set of values; $k_{e f}[-]$ - effective partitioning coefficient of the volume element between the solid and the liquid states; $g_{s}[-]-$ solid fraction; and $\alpha$ [-] - the dimensionless Fourier number for the mass transfer, see Eq. (5). The quantity $n$ is of a statistical nature and it expresses the percentage of the measured values, which can be found within the interval $C_{0} \pm$ $n s_{C}$ (where $s_{C}$ is the standard deviation of the measured concentration of the set of values). If $n=2$, then $95 \%$ of all measured values can be found within this interval.

If the dimensionless parameter $\alpha$ is known for each oxide, then a key exists for the clarification of the relation between the local solidification time $\theta$ of EUCOR and the diffusion coefficient $D$ of the relevant oxide within the solidifying phase, and the structure parameter $L$, which characterises the distances between individual dendrites. The equation of the dimensionless parameter $\alpha$ is as follows

$$
\alpha=D \theta / L^{2}
$$

\subsection{Application of the chemical heterogeneity model}

The verification of the possibility of combining both methods was conducted with samples taken from the EUCOR blocks - from the edge (sample B) - and from the centre underneath the riser (sample $\mathrm{C}$ ).

Both the measured and computed parameters of chemical heterogeneity and the computed parameters of the local solidification time $\theta$ were calculated according to the temperature-field model. The calculated local solidification time of the sample $B$ was $\theta_{B}=112.18$ [s] and of sample $C$ was $\theta_{C}=283.30$ [s].

The computed values of the parameter $\alpha$ and the local solidification time $\theta$ determine, via their ratio, the quotient of the diffusion coefficient $D$ and the square of the structure parameter $L$, which means that the following relation applied:

$$
\alpha / \theta=D / L^{2} \quad[1 / s]
$$

The calculated values of Eq. (6) for oxides of the samples B and C are arranged in the following Table 1, together with the parameters $\alpha$.

The parameter $\alpha / \theta=D / L^{2}$ calculated for the oxide of zirconium and hafnium differs significantly from the parameter calculated for other oxides. This could be explained by the fact that zirconium contains hafnium as an additive and they therefore segregate together, and the forming oxides of zirconium and hafnium have the highest melting temperature. Both oxides segregate first from the melt, already in the solid state. Further redistribution of the oxides of both elements runs on the interface of the remaining melt and other oxides segregate afterwards only in a very limited extent. It is therefore possible to count on the fact that the diffusion coefficients of zirconium and hafnium in the successively forming crystallites are very small (i.e. $D_{\mathrm{Zr}} \rightarrow 0$ a $D_{\mathrm{Hf}} \rightarrow 0$ ). On the other hand, the very 
close values of the parameters $\alpha / \theta=D / L^{2}$ of the remaining seven analysed oxides, $D / L_{\mathrm{B}}{ }^{2}=(6.51 \pm 0.25) \cdot 10^{-4}$ and $D / L_{\mathrm{C}}{ }^{2}=(2.45 \pm 0.12) \cdot 10^{-4}[1 / \mathrm{s}]$, indicate that the redistribution of these oxides between the melt and the solid state runs in the way similar to that in metal alloys, namely steels.

Table 1: Calculated values of the parameter $\alpha$ and values calculated by equation (4).

\begin{tabular}{||c||c|c|c|c||}
\hline \hline Oxide & $\begin{array}{c}\text { Sample B: } \\
\boldsymbol{\alpha}\end{array}$ & $\begin{array}{c}\alpha / \theta_{\mathrm{B}} \cdot 10^{4} \\
{[1 / \mathrm{s}]}\end{array}$ & $\begin{array}{c}\text { Sample C: } \\
\boldsymbol{\alpha}\end{array}$ & $\begin{array}{c}\alpha / \theta_{\mathrm{C}} \cdot 10^{4} \\
{[1 / \mathrm{s}]}\end{array}$ \\
\hline \hline $\mathrm{Na}_{2} \mathrm{O}$ & 0.0732 & 6.53 & 0.0691 & 2.44 \\
\hline $\mathrm{Al}_{2} \mathrm{O}_{3}$ & 0.0674 & 6.01 & 0.0662 & 2.34 \\
\hline $\mathrm{SiO}_{2}$ & 0.0741 & 6.61 & 0.0663 & 2.34 \\
\hline $\mathrm{ZrO}_{2}$ & 0.00035 & 0.0312 & 0.00008 & 0.0028 \\
\hline $\mathrm{K}_{2} \mathrm{O}$ & 0.0721 & 6.43 & 0.0665 & 2.35 \\
\hline $\mathrm{CaO}$ & 0.075 & 6.69 & 0.0703 & 2.48 \\
\hline $\mathrm{TiO}_{2}$ & 0.0759 & 6.77 & 0.0757 & 2.67 \\
\hline $\mathrm{Fe}_{2} \mathrm{O}_{3}$ & 0.0732 & 6.53 & 0.0711 & 2.51 \\
\hline $\mathrm{HfO}_{2}$ & 0.0165 & 1.47 & 0.00017 & 0.006 \\
\hline \hline
\end{tabular}

For the calculation it is possible to use - in the first approximation - the diffusion coefficients of the oxides in the slag at temperatures of $1765^{\circ} \mathrm{C}$ (solidus) and $1775^{\circ} \mathrm{C}$ (liquidus), and the average value of $(2.07 \pm 0.11) \cdot 10^{-6} \mathrm{~cm}^{2} / \mathrm{s}$ (the data refer to the diffusion of aluminium in the slag of the composition of $39 \% \mathrm{CaO}, 20 \% \mathrm{Al}_{2} \mathrm{O}_{3}$ and $41 \% \mathrm{SiO}_{2}$ [5]). In these cases, and using Eq. (6), it is possible to obtain the magnitude of the structure parameters that govern the chemical heterogeneity of the values:

$$
\begin{aligned}
& L_{B}=\sqrt{\left(2.07 \cdot 10^{-6}\right) /\left(6.51 \cdot 10^{-4}\right)}=0.5639 \cdot 10^{-3} \quad[\mathrm{~m}] \\
& \text { and } L_{C}=\sqrt{\left(2.07 \cdot 10^{-6}\right) /\left(2.45 \cdot 10^{-4}\right)}=0.9192 \cdot 10^{-3} \quad[\mathrm{~m}],
\end{aligned}
$$

which corresponds to $564 \mu \mathrm{m}$ in the sample B and $919 \mu \mathrm{m}$ in the sample C.

The comparison of the micro-structures in Figs. 3 and 4 clearly shows that the micro-structure of the sample B is significantly finer than the micro-structure of the sample $\mathrm{C}$. This fact corresponds to the calculated structure parameters $L_{B}$ and $L_{C}$ obtained on the basis of data from both models.

\subsection{Consequences of the chemical heterogeneity model}

The character of the structure in Fig. 3 shows that the cooling rate for sample B was higher than for sample C (Fig. 4). From these two figures, it is possible to see that the higher value of the structure parameter $L$ corresponds with the lower cooling rate related to the same material. Both structure parameters $L_{B}$ and $L_{C}$ 
were calculated using the combination of the two models (the numerical model of the temperature field and the model of the chemical heterogeneity). Numerical model of the temperature field of casting provides information about the local solidification time at any point of the casting. It means that it provides also the values of the local solidification time for any critical point of the casting, which has the tendency to cracking or to fracture. The chemical heterogeneity model enables the estimation of the structure parameters at this critical point of castings.

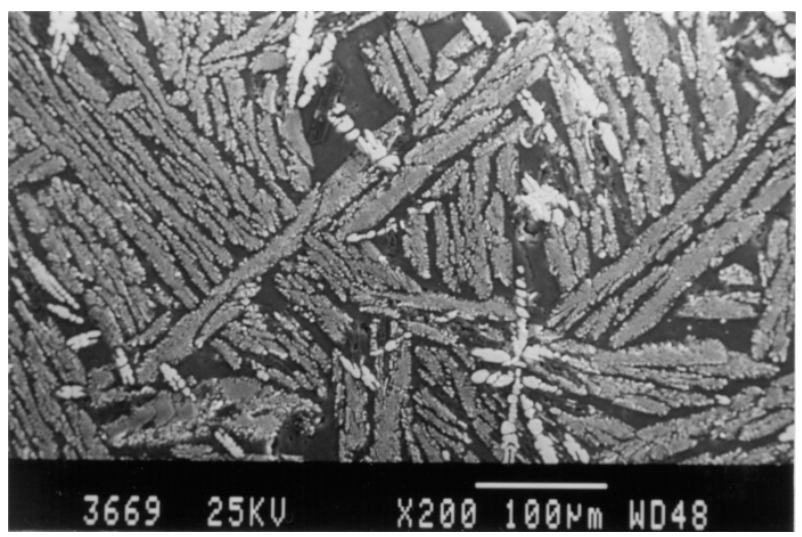

Figure 3: $\quad$ The structure of the sample $B\left(\mathrm{~L}_{\mathrm{B}}=564 \mu \mathrm{m}\right)$.

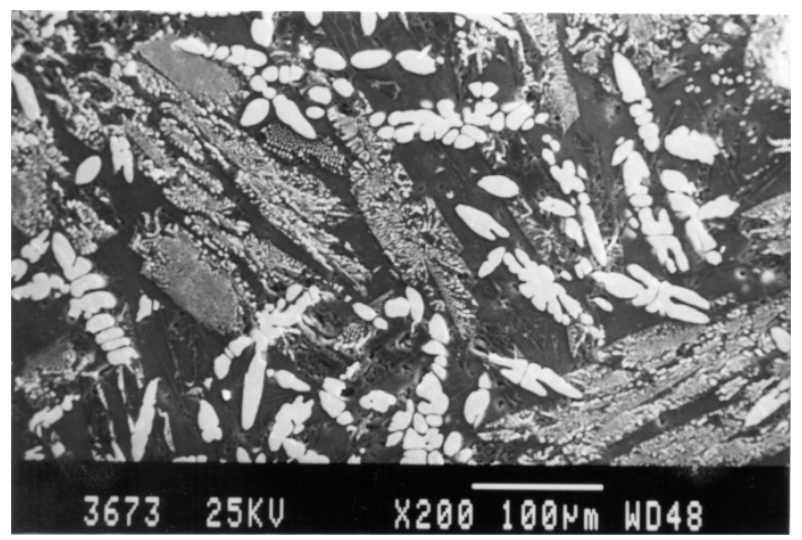

Figure 4: $\quad$ The structure of the sample $\mathrm{C}\left(\mathrm{L}_{\mathrm{C}}=919 \mu \mathrm{m}\right)$.

It is possible to use the equation derived from the fracture mechanics in the following form:

$$
K_{I C} \geq \sigma_{B} \sqrt{\pi L_{B}}=\sigma_{C} \sqrt{\pi L_{C}}
$$


where $K_{I C}$ is the fracture toughness $\left[\mathrm{MPa} . \mathrm{m}^{1 / 2}\right], \sigma_{\mathrm{B}}, \sigma_{\mathrm{C}}$ are the strains on the tips of structure defects $[\mathrm{MPa}]$, and $L_{B}$ and $L_{C}$ are the diameters of the structure parameters (cells) in the samples B and C [m]. High probability of the creation of primary technological defects in the castings (the micro-shrinkage porosity, micro-shrinkage cavity, etc.) on their grains exists. It follows from Eq. 9 that

$$
\left(\frac{\sigma_{B}}{\sigma_{C}}\right)^{2}=\frac{L_{C}}{L_{B}}=\frac{919.2}{563.9}=1.63
$$

It means that the relation between the structure parameters $L_{B}$ and $L_{C}$ expresses the relation between $\sigma_{B}$ and $\sigma_{C}$ strains on the tips of potential structure defects of the samples B and C. At the same fracture toughness, the strain on the sample B can be slightly higher when compared with the strain on the sample C.

\section{Discussion and conclusions}

The investigation of the temperature field had the following two objectives:

- A directed solidification as the primary condition for a healthy casting,

- The optimisation of the casting technology, together with the preservation of the optimum utility properties of the product.

The achievement of these objectives depended on the ability to analyse and consequently to control the effects of the main factors which characterise or accompany the solidification process.

The results of the research should reveal the causes of heterogeneities in the casting with respect to the phase and the structural changes. It should also focus on the thermo-kinetics of the formation of shrinkage porosities and cavities. In addition, attention should also be given to the control of optimisation of the shape and the sizes of the risers, the method of insulation, the treatment of the level, etc. The main economic criteria focus on the saving of liquid material, mould and insulation materials, as well as energy, and on the optimisation of the casting process and the properties of the cast products.

The paper presents results of the pilot computation of the temperature field of the ceramic material EUCOR. These results prove that with the use of the numerical model it is possible to optimise the technology of production of this material, and to enhance in this way its utilisation in a number of industries, namely in the glass-making, power-generating, foundry and metallurgical industries.

This paper discusses the numerical model of the transient temperature field and the numerical model of the chemical heterogeneity, their application and combination. Combining of both models makes it possible to estimate the structure parameter, which expresses the size of the crystallites in the resulting material structure. 


\section{Acknowledgements}

This paper was prepared thanks to the work conducted within the projects supported by the Czech Scientific Foundation (Nos. 106/08/0606, 106/09/0940, 106/09/0969, and P107/11/1566).

\section{References}

[1] Chvorinov, N. Crystallization and non-homogeneity of steel. Czechoslovak Academy of Science Publishing Company, Prague, 1954.

[2] Kavička, F. et al. Castings from corundo-baddeleyite ceramics - their properties and utilization for wear resistant materials, piping, etc. (in Czech). Slévárenství 49 (2001) 9, pp. 524-529.

[3] Dobrovská, J. et. al. Possible ways of prediction of the distribution curves of dendritic segregation of alloying elements in steels. Scripta Mater., 18 (1998) 10, pp. 1583-1598.

[4] Brody, H. D., Flemings, M. C. Solute redistribution in dendritic solidification. Trans. AIME, 1966, vol. 236, p. 615-624.

[5] Elliott, J. F., Gleiser, M., Ramakrishna, V. Thermochemistry for steelmaking. AISI, Reading, Massachusetts, U.S.A., London, England, 1963. 